\title{
SEQUENTIAL PROBABILITY RATIO TEST FOR COLLISION AVOIDANCE MANEUVER DECISIONS
}

\author{
J. Russell Carpenter*and F. Landis Markley ${ }^{\dagger}$
}

\begin{abstract}
When facing a conjunction between space objects, decision makers must chose whether to maneuver for collision avoidance or not. We apply a well-known decision procedure, the sequential probability ratio test, to this problem. We propose two approaches to the problem solution, one based on a frequentist method, and the other on a Bayesian method. The frequentist method does not require any prior knowledge concerning the conjunction, while the Bayesian method assumes knowledge of prior probability densities. Our results show that both methods achieve desired missed detection rates, but the frequentist method's false alarm performance is inferior to the Bayesian method's.
\end{abstract}

\section{INTRODUCTION}

We often hear it said that "space is big" by which we think decision makers mean that collisions between space objects are so rare that many collision avoidance maneuver (CAM) decisions are the result of false alarms. Nevertheless, the consequences of a missed detection might be catastrophic. It is our understanding of current CAM decision procedures that they do not explicitly account for the costs and risks of false alarms and missed detections. It seems to us that a utilitarian decision making procedure that balances the costs and risks of false alarms and missed detections would provide some benefit to decision makers. We propose herein such a procedure that is applicable to a common class of CAM decision problems.

The remainder of this paper is organized as follows. In the next section, we review how the full CAM decision problem reduces under certain assumptions to the comparison of hypotheses concerning parameters of Rician densities; assumptions on which at present, our proposed method depends. Then, we briefly discuss collision avoidance as a decision problem involving equalizing utilities based on cost and risk. Next, we review Wald's Sequential Probability Ratio Test (SPRT), ${ }^{1}$ which forms the basis of our proposed method. While the SPRT involves a likelihood ratio between two simple hypotheses, the CAM decision problem as we define it involves a comparison among two continuous sets of hypotheses. In the subsequent section, which we believe involves the main technical contribution of this paper, we discuss two methods to reduce the compound hypotheses to simple ones. In the penultimate section, we illustrate and compare our methods using a static example based on the Magnetospheric Multi-Scale (MMS) mission, and finally we conclude with a summary and suggestions for follow-on work.

\footnotetext{
*Aerospace Engineer, Navigation and Mission Design Branch, NASA Goddard Space Flight Center, Code 595, Greenbelt, MD 20771.

${ }^{\dagger}$ Aerospace Engineer, Attitude Control Systems Engineering Branch, NASA Goddard Space Flight Center, Code 591, Greenbelt, MD 20771.
} 


\section{PROBLEM STATEMENT}

\section{The Simplified CAM decision problem}

Our approach to the CAM decision problem starts with the ideas of Foster and Estes. ${ }^{2}$ In their study of the need for CAMs by the International Space Station, they showed that under conditions typical of many conjunctions, only the projection of the three-dimensional relative position error ellipsoid onto the conjunction plane at the time of closest approach need be considered when computing the probability of collision. Akella and Alfriend ${ }^{3}$ later showed that a more general problem explicitly considering collision probability as a function of time throughout the conjunction reduces under certain conditions to the static problem considered by Foster and Estes. Chan ${ }^{4}$ later showed that in many cases, the problem could be further simplified to that of evaluating Rician densities. ${ }^{5}$ In this section, we summarize the results of the cited works.

Let $\left\{\boldsymbol{R}_{i}, \boldsymbol{V}_{i}\right\}, i=1,2$ represent the inertial position and velocity of two space objects. Then, the relative position and velocity between the objects in a frame fixed to the orbital velocity and orbit normal vectors of object 1 are

$$
\begin{aligned}
\boldsymbol{r} & =\boldsymbol{R}_{2}-\boldsymbol{R}_{1} \\
\boldsymbol{v} & =\boldsymbol{V}_{2}-\boldsymbol{V}_{1}-\boldsymbol{\omega}_{1} \times \boldsymbol{r}
\end{aligned}
$$

where $\omega_{1}$ is the instantaneous orbital angular velocity of object 1 . The relative position is uncertain due to knowledge errors, $e$,

$$
\boldsymbol{r}=\overline{\boldsymbol{r}}+\boldsymbol{e}
$$

where $\bar{r}=\mathrm{E}[\boldsymbol{r}]$ is the nominal relative position vector. The associated error covariance matrix is

$$
\begin{aligned}
P & =\mathrm{E}\left[(\boldsymbol{r}-\overline{\boldsymbol{r}})(\boldsymbol{r}-\overline{\boldsymbol{r}})^{\top}\right] \\
& =P_{R 1}+P_{R 2}-P_{R 1 R 2}-P_{R 1 R 2}^{\mathrm{\top}}
\end{aligned}
$$

where

$$
\begin{aligned}
P_{R i} & =\mathrm{E}\left[\left(\boldsymbol{R}_{i}-\mathrm{E}\left[\boldsymbol{R}_{i}\right]\right)\left(\boldsymbol{R}_{i}-\mathrm{E}\left[\boldsymbol{R}_{i}\right]\right)^{\top}\right] \\
P_{R i R j} & =\mathrm{E}\left[\left(\boldsymbol{R}_{i}-\mathrm{E}\left[\boldsymbol{R}_{i}\right]\right)\left(\boldsymbol{R}_{j}-\mathrm{E}\left[\boldsymbol{R}_{j}\right]\right)^{\top}\right]
\end{aligned}
$$

The cross-covariance matrix $P_{R i R j}$ is often negligible unless common measurements such as ranges between the objects have been used in the estimation process.

Figure 1 depicts a dangerously close approach between two satellites flying in formation. The large red ellipsoid in the figure represents the relative position knowledge uncertainty, which is the propagation of post-maneuver state error uncertainty over approximately half an orbit period. One can see from the figure that the largest component of the knowledge uncertainty is generally along the velocity direction; however, there is a significant projection of the uncertainty into the conjunction plane, i.e. the plane containing the relative position vector at the time of closest approach. The figure shows this projection as a blue empty ellipse in the isometric projection on the left. On the right, the figure shows the view normal to the conjunction plane, and the overlap between the knowledge error ellipse ("one sigma") and the green sphere enclosing the combined hard-body radius of the two spacecraft.

For such encounters, the probability of collision at the time of closest approach is the triple integral over the hard-body volume of the density function associated with the knowledge error. The key 

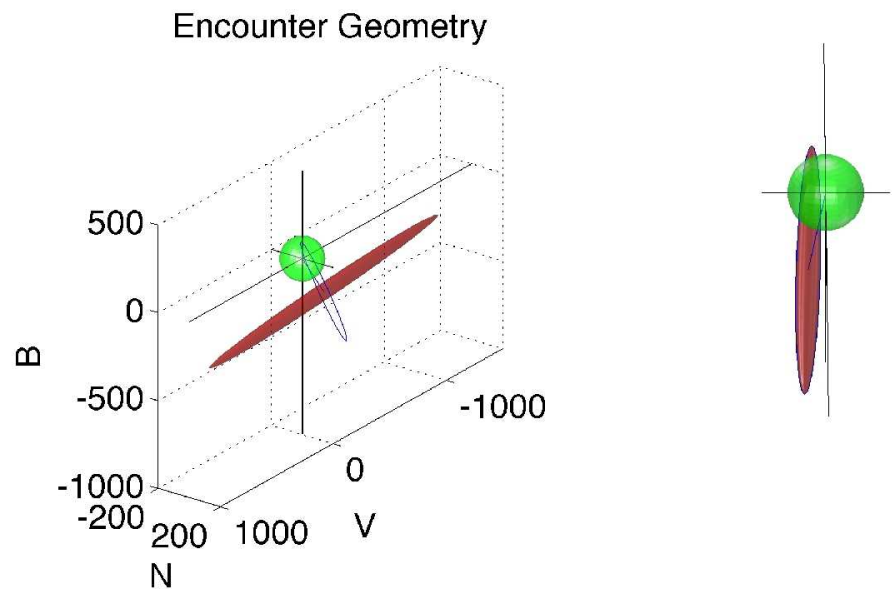

Figure 1 Stress-case simulated conjunction geometry for two MMS satellites near perigee. The green sphere encloses the combined tip-to-tip radius $(120 \mathrm{~m})$ of the two satellites. The red ellipsoid represents the relative position knowledge error covariance. The $V N B$ coordinate frame on the left is aligned with the orbit velocity, orbit normal, and orbit bi-normal directions at the time of time of closest approach, and the view on the right projects the encounter into the conjunction plane. Units are meters.

simplifying insight of Foster and Estes, ${ }^{2}$ which was clarified and refined by Akella and Alfriend, ${ }^{3}$ is that the probability of collision reduces to an integration only within the conjunction plane, under certain conditions. Akella and Alfriend ${ }^{3}$ specify the conditions under which this simplification holds during the short interval of the encounter, as follows:

1. The trajectories of the objects are straight lines.

2. Velocity uncertainty integrates into negligible position uncertainty, and consequently the position uncertainty is constant.

3. The knowledge error density is Gaussian.

Figure 2 illustrates the procedure whereby $\mathrm{Chan}^{4}$ converted the double integral over Gaussian densities in the conjunction plane into a single integral of a Rician density governing the scalar miss distance. As Papoulis ${ }^{5}$ describes, a Rician sample can be viewed as the root-sum-square of two samples drawn from Gaussian densities with the same mean and variance. Viewing the Gaussian samples as coordinates in a plane, the Rician sample can be viewed as a polar radius coordinate; the corresponding angle coordinate is free. As Figure 2 shows, Chan's idea is to re-scale the coordinates of the conjunction plane so as to circularize the knowledge error ellipse. Now, the re-scaled miss distance has an isotropic density, and the Rician density applies. However, the region of integration, i.e. the hard body disk, is now elliptical, making its evaluation more difficult. To avoid this complication, Chan suggests that we replace the now-elliptical hard body region with a circle of equivalent area. The result is that the probability of collision, $P_{c}$, is approximated by

$$
P_{c}=\int_{0}^{R} \frac{z}{\sigma^{2}} \exp \left(-\frac{z^{2}+\nu^{2}}{2 \sigma^{2}}\right) I_{o}\left(\frac{z \nu}{\sigma^{2}}\right) \mathrm{d} z \triangleq \int_{0}^{R} \operatorname{ric}(z \mid \nu, \sigma) \mathrm{d} z
$$


where $R$ represents the re-scaled hard-body radius, and $I_{o}$ denotes the modified Bessel function of the first kind, of order zero. We identify the Rician's non-centrality parameter, $\nu$, with the true re-scaled miss distance, and view samples drawn from the Rician as representing observations of the re-scaled predicted miss distance. Figure 3 depicts two examples of the Rician density function.
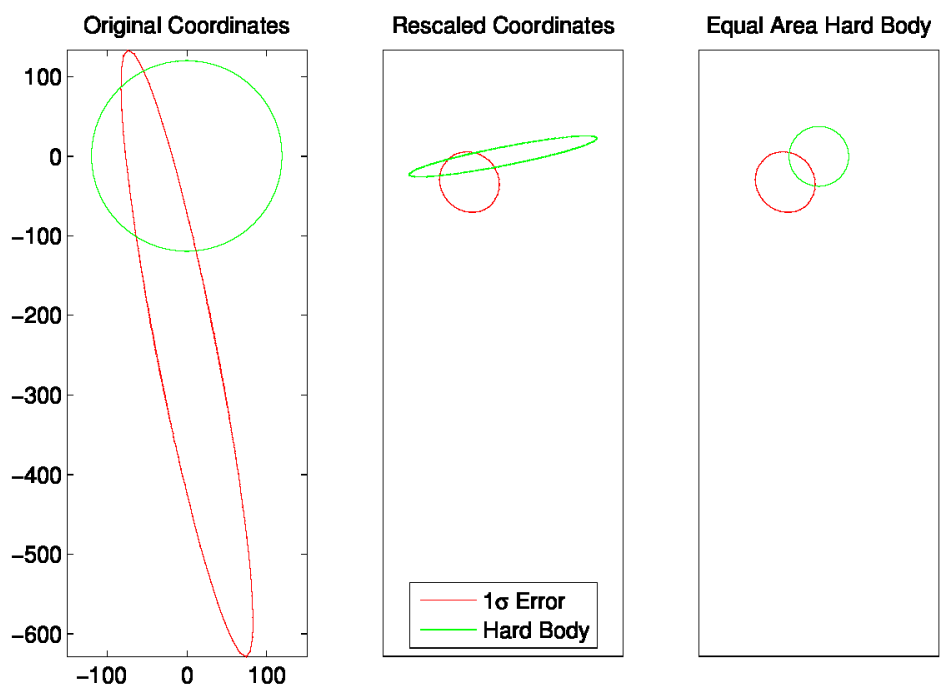

Figure 2 Conversion to Rician Problem. The left pane shows the conjunction geometry in the original coordinates (meters); in the middle pane, the coordinates have been compressed along the major axis of the knowledge error ellipse so as to circularize it, and the hard-body sphere has therefore been squashed into an ellipse; in the final pane, the elliptical hard-body has been replaced by a circle of equivalent area.

\section{CAM as a Decision Problem}

Assume that we receive a sequence of independent observations $\mathcal{Z}_{n}=\left\{z_{1}, z_{2}, \ldots, z_{n}\right\}$ of the re-scaled miss distance, each with known re-scaled variance, $\sigma_{i}^{2}$. We wish to design a procedure that will use the observations to inform a decision concerning the need for a collision avoidance maneuver. In this procedure, we will compare two hypotheses, $H_{0}$ and $H_{1}$. The null hypothesis, $H_{0}$, is that the miss distance is unsafe; that is, that the true re-scaled miss distance, $\nu$, is less than or equal to the re-scaled hard-body radius, $R$. The alternative hypothesis, $H_{1}$, is that $\nu>R$. Figure 4 illustrates the two hypotheses as families of Rician densities.

Since we have to make this decision prior to the time of closest approach, based on a prediction of the miss distance, there are four possible outcomes:

1. A correct decision to maneuver: our miss distance prediction indicated an unsafe conjunction, and an unsafe conjunction really would have occurred. We will call this condition a true alarm, or a true positive, and denote the associated probability of detection as $P_{d}$.

2. An incorrect decision to maneuver: our miss distance prediction indicated an unsafe conjunction, but the miss distance was actually large enough to be safe. We will call this condition a false alarm, or a false positive, and denote the associated probability of false alarm as $P_{f a}$. This type of error creates a nuisance, but is not catastrophic. 


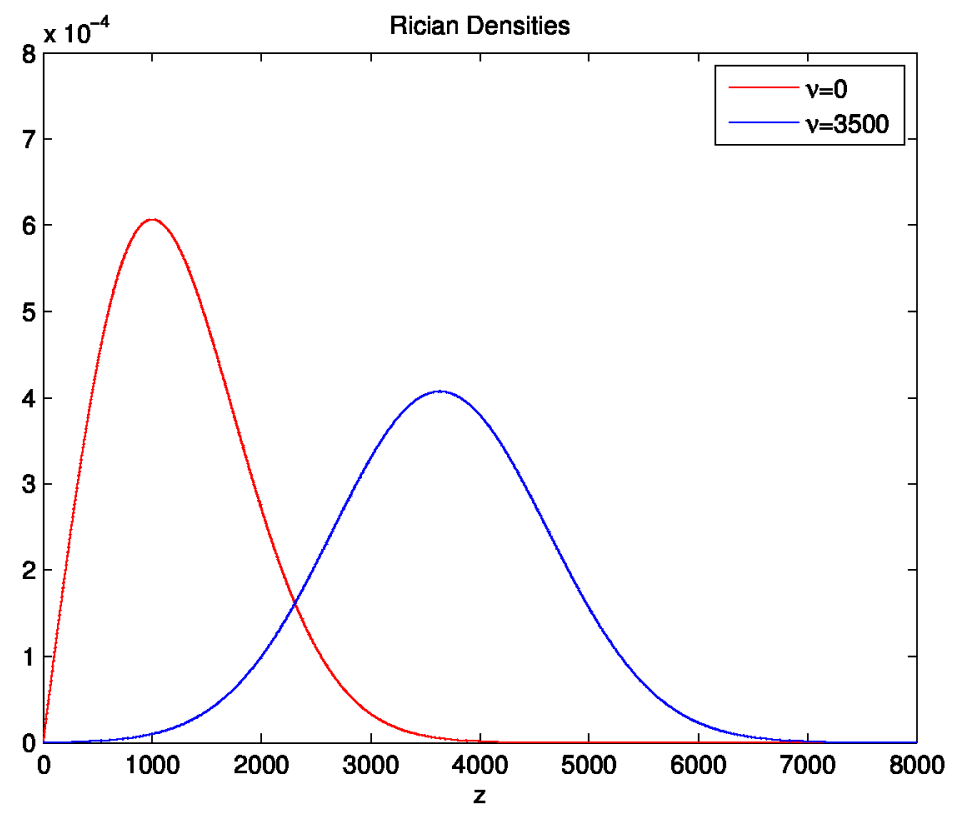

Figure 3 Two examples of Rician densities, with $\sigma=1000$. For large $\nu$, the Rician begins to resemble a Gaussian.

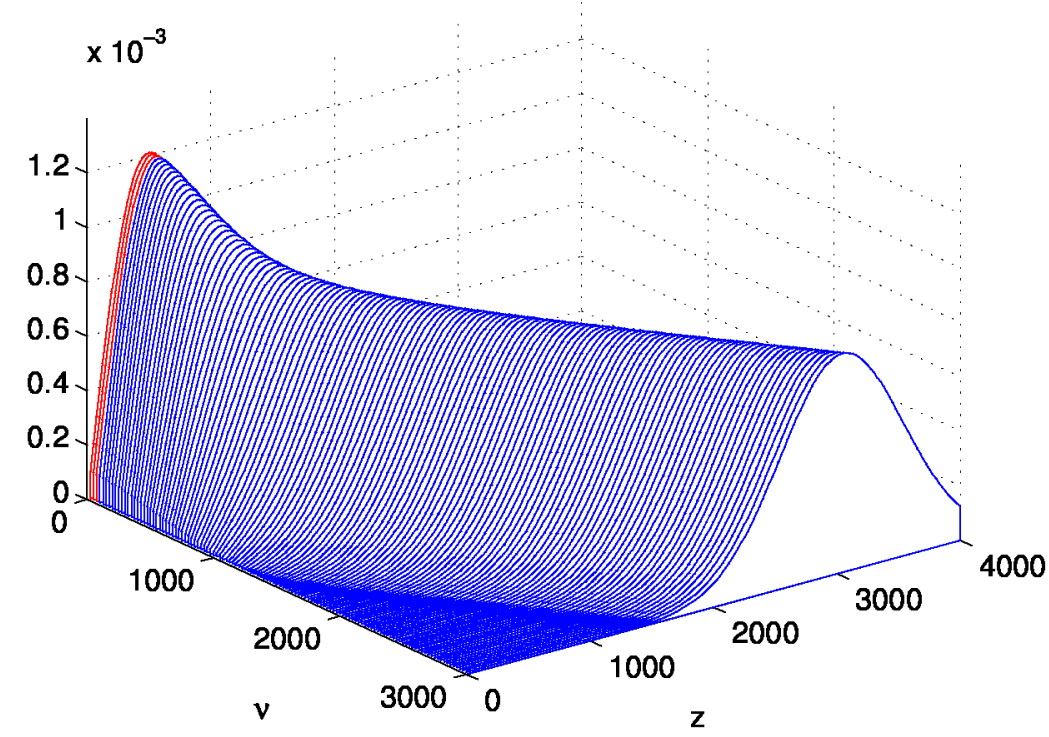

Figure 4 Families of Rician Densities for $\sigma=500$. The red Ricians correspond to $H_{0}: \nu \leq R$, and the blue Ricians to $H_{1}: \nu>R$, where $R=50$. 
3. A correct decision not to maneuver: our predicted miss distance was large enough to be safe, and the actual miss distance was also large enough to be safe. We will call this condition a true dismissal, or a true negative, and denote the associated probability of rejection as $P_{r}$.

4. An incorrect decision not to maneuver: our predicted miss distance was large enough to be safe, but the actual miss distance was small enough to be unsafe. We will call this condition a missed detection, or a false negative, and denote the associated probability of missed detection as $P_{m d}$. This type of error may be catastrophic.

In principle, it should be possible to determine the cost of performing a CAM, $c_{m}$, and it is also possible to determine the value of the objects involved in the conjunction, $V$. Thus, it is possible to associate a cost with each of the outcomes just described. Table 1 illustrates the mapping of decisions and conditions to outcomes and decision costs. Here, we have assumed that the cost of a rejection is minimal, although perhaps not zero, since there may be a cost associated with CAM operations regardless of whether a maneuver occurs. The expected cost of each outcome is therefore the product of the cost each time it occurs, and the probability with which it might occur.

Table 1. Decision Matrix.

\begin{tabular}{|c|c|c|c|}
\hline & \multicolumn{2}{|c|}{ Decision } \\
\hline & & Accept $H_{0} \&$ maneuver & Reject $H_{0} \&$ dismiss \\
\hline \multirow{2}{*}{ : } & $\begin{array}{l}H_{0} \text { is true: } \\
\text { collision } \\
\text { will occur }\end{array}$ & $\begin{array}{l}\text { True Positive: } \\
\text { Probability }=P_{d}=1-P_{m d} \\
\text { Cost }=c_{m}\end{array}$ & $\begin{array}{l}\text { Missed Detection: } \\
\text { Probability }=P_{m d} \\
\text { Cost }=V\end{array}$ \\
\hline & $\begin{array}{l}H_{0} \text { is false: } \\
\text { trajectory } \\
\text { is safe }\end{array}$ & $\begin{array}{l}\text { False Alarm: } \\
\text { Probability }=P_{f a} \\
\text { Cost }=c_{m}\end{array}$ & $\begin{array}{l}\text { True Negative: } \\
\text { Probability }=P_{r}=1-P_{f a} \\
\text { Cost }=\epsilon\end{array}$ \\
\hline
\end{tabular}

Since the probabilities that $H_{0}$ is true, and false, are $P_{c}$, and $1-P_{c}$, respectively, the total expected cost, $c$, of employing this decision procedure is

$$
\mathrm{E}[c]=P_{c}\left[\left(1-P_{m d}\right) c_{m}+P_{m d} V\right]+\left(1-P_{c}\right)\left[P_{f a} c_{m}+\left(1-P_{f a}\right) \epsilon\right]
$$

Neyman-Pearson theory ${ }^{6}$ shows that for any particular fixed number of observations, there exists a Pareto optimal relationship between $P_{m d}$ and $P_{f a}$; that is, if for example one fixes $P_{m d}=P_{m d}^{*}$, then there exists a corresponding minimal $P_{f a}^{*}\left(P_{m d}^{*}\right)$. Letting $P_{f a}^{* *}=\partial P_{f a}^{*} / \partial P_{m d}^{*}$, optimization of Eq. 9 shows that the minimal cost occurs when

$$
P_{f a}^{\prime *}=-\frac{P_{c}}{\left(1-P_{c}\right)} \frac{\left(V-c_{m}\right)}{\left(c_{m}-\epsilon\right)} \approx-P_{c} \frac{V}{c_{m}}
$$

Since in general, one cannot arbitrarily decrease $P_{m d}$ without increasing $P_{f a}$, we can assume that $P_{f a}^{*}\left(P_{m d}^{*}\right)$ has the form

$$
P_{f a}^{*}\left(P_{m d}^{*}\right) \approx-k / P_{m d}^{*}
$$

where $k$ is some given constant; this implies that $P_{f a}^{\prime *} \approx P_{f a}^{*} / P_{m d}^{*}$. Combining this with Eq. 10 results in the approximation that

$$
P_{c} P_{m d} V \approx P_{f a} c_{m}\left(1-P_{c}\right)
$$

which shows that we minimize CAM costs by choosing $P_{m d}$ and $P_{f a}$ such that the expected costs of missed detection will approximately balance the costs of false alarms. 


\section{WALD'S SPRT}

Let $f\left(\mathcal{Z}_{n} \mid H_{0}\right)$ be the probability density associated with having obtained the set of sequential observations $\mathcal{Z}_{n}$ given that the null hypothesis, i.e. that the miss distance is unsafe, were true* Let $f\left(\mathcal{Z}_{n} \mid H_{1}\right)$ be the probability density associated with obtaining the same set of observations given that the alternative hypothesis, i.e. that the miss distance is safe, were true. Wald's SPRT compares the ratio of these probabilities to thresholds related to the false alarm and missed detection probabilities, as follows. ${ }^{1}$

Define the likelihood ratio as:

$$
\Lambda_{n}=\frac{f\left(\mathcal{Z}_{n} \mid H_{1}\right)}{f\left(\mathcal{Z}_{n} \mid H_{0}\right)}=\frac{f\left(\mathcal{Z}_{n} \mid \nu>R\right)}{f\left(\mathcal{Z}_{n} \mid \nu \leq R\right)}
$$

Wald recommends that we establish decision limits $A$ and $B$ such that whenever $B<\Lambda_{n}<A$ we will, if possible, seek another observation. If $\Lambda_{n} \leq B$, then we will accept the null hypothesis and maneuver. If $\Lambda_{n} \geq A$, then we will accept the alternative hypothesis and dismiss the alarm. Wald's explanations for the thresholds $A$ and $B$ are that we will accept the alternative hypothesis if it is $A$ times more likely than the null, and accept the null hypothesis if it is $1 / B$ times more likely than the alternative. Wald shows that such a procedure will terminate with probability one, and that

$$
A \leq \frac{1-P_{f a}}{P_{m d}} \text { and } B \geq \frac{P_{f a}}{1-P_{m d}}
$$

which implies that the $P_{f a}$ and $P_{m d}$ resulting from such a procedure will be constrained by

$$
\begin{aligned}
& P_{f a} \leq B\left(1-P_{m d}\right) \\
& P_{f a} \leq 1-P_{m d} A
\end{aligned}
$$

Note that unlike a Neyman-Pearson test which employs a fixed number of observations, the number of observations required for the SPRT to terminate is a random variable.

To see how we could apply Wald's procedure to the CAM decision problem, suppose we knew that the re-scaled true miss distance, $\nu$, could take on only two definite values: $\nu_{0}=0$ and $\nu_{1}=6 R$. Then, our hypotheses would be $H_{0}: \nu=\nu_{0}=0$, and $H_{1}: \nu=\nu_{1}=6 R$. Since the observations $\mathcal{Z}_{n}$ are independent, Eq. 13 becomes

$$
\Lambda_{n}=\frac{f\left(\mathcal{Z}_{n} \mid \nu=\nu_{1}\right)}{f\left(\mathcal{Z}_{n} \mid \nu=\nu_{0}\right)}=\frac{\prod_{i=1}^{n} \operatorname{ric}\left(z_{i} \mid \nu_{1}, \sigma_{i}\right)}{\prod_{i=1}^{n} \operatorname{ric}\left(z_{i} \mid \nu_{0}, \sigma_{i}\right)}
$$

from which we can see that if the $z_{i}$ are more consistent with $\nu_{1}$ than with $\nu_{0}$, the product in the numerator of Eq. 17 will tend to dominate the product in its denominator, and the test will terminate with $\Lambda_{n}>A$, and vice versa. Wald points out that it may be numerically superior to use the log-likelihood ratio, rather than Eq. 17,

$$
\log \Lambda_{n}=\sum_{i=1}^{n} \log \operatorname{ric}\left(z_{i} \mid \nu_{1}, \sigma_{i}\right)-\sum_{i=1}^{n} \log \operatorname{ric}\left(z_{i} \mid \nu_{0}, \sigma_{i}\right)
$$

and we employ this suggestion where applicable below.

\footnotetext{
${ }^{*}$ Here, and subsequently, we use the function $f(\cdot)$ to refer to a generic probability density function.
} 
In reality, we do not have simple hypotheses of this sort; so, in order to make use of Wald's procedure, we must reduce the compound hypotheses $H_{0}: \nu \leq R$, and $H_{1}: \nu>R$, which Figure 4 illustrates, to simple hypotheses. That is, we must reduce the family of red Ricians in Figure 4 to a single hypothesis that we can use in the denominator of Eq. 13, and reduce the family of blue Ricians in Figure 4 to a single hypothesis that we can use in the numerator of Eq. 13. In the next two subsections, we propose a frequentist and a Bayesian method to accomplish this reduction.

\section{Frequentist Approach}

Suppose we find the $\nu_{1} \in(R, \infty)$ that maximizes the product in the numerator of Eq. 17, and also the $\nu_{0} \in[0, R]$ that maximizes the product in the denominator of Eq. 17. Using these maximum likelihood estimates, $\hat{\nu}_{1}$ and $\hat{\nu}_{0}$, respectively, in each region would accomplish our goal of reducing the compound hypotheses $H_{0}: \nu \leq R$ and $H_{1}: \nu>R$ to the simple hypotheses $H_{0}: \nu=\hat{\nu}_{0}$ and $H_{1}: \nu=\hat{\nu}_{1}$. A simple, bounded, line search will produce such estimates.

Next, we need to determine the decision ratios $A$ and $B$. Suppose we define critical measurement thresholds, $z_{A}$ and $z_{B}$, such that we would accept the alternative hypothesis, $\nu>R$, if a single measurement $z_{1}$ exceeds $z_{A}$, and we would accept the null hypothesis, $\nu \leq R$, if $z_{1} \leq z_{B}$. We would want $z_{A}$ to be large enough that the probability is $P_{m d}$ or less that the null hypothesis were true when $z_{1}>z_{A}$. Refer to the upper pane of Figure 5 for an illustration of this idea. Thus,

$$
z_{A}=\max _{\nu \in[0, R]} \operatorname{ric}^{-1}\left(1-P_{m d} \mid \nu, \sigma_{1}\right)
$$

where

$$
z_{*}=\operatorname{ric}^{-1}(P \mid \nu, \sigma)=f(P): P-\int_{0}^{z_{*}} \operatorname{ric}(z \mid \nu, \sigma) \mathrm{d} z=0
$$

Similarly, we would want $z_{B}$ to be small enough that the probability is $P_{f a}$ or less that the alternative hypothesis were true when $z_{1}<z_{B}$, as the lower pane of Figure 5 depicts. Thus,

$$
z_{B}=\max _{\nu \in(R, \infty)} \operatorname{ric}^{-1}\left(P_{f a} \mid \nu, \sigma_{1}\right)
$$

Then, to find $A$ and $B$ we merely evaluate Eq. 17 at $z_{A}$ and $z_{B}$ respectively:

$$
\begin{aligned}
A^{F} & =\frac{\max _{\nu \in(R, \infty)} \operatorname{ric}\left(z_{A} \mid \nu, \sigma_{1}\right)}{\max _{\nu \in[0, R]} \operatorname{ric}\left(z_{A} \mid \nu, \sigma_{1}\right)} \\
B^{F} & =\frac{\max _{\nu \in(R, \infty)} \operatorname{ric}\left(z_{B} \mid \nu, \sigma_{1}\right)}{\max _{\nu \in[0, R]} \operatorname{ric}\left(z_{B} \mid \nu, \sigma_{1}\right)}
\end{aligned}
$$

Thus, the frequentist version of our decision procedure consists of the following methodology. As each predicted re-scaled miss distance becomes available, accumulate the log-likelihood ratio:

$$
\log \Lambda_{n}^{F}=\max _{\nu \in(R, \infty)} \sum_{i=1}^{n} \log \operatorname{ric}\left(z_{i} \mid \nu, \sigma_{i}\right)-\max _{\nu \in[0, R]} \sum_{i=1}^{n} \log \operatorname{ric}\left(z_{i} \mid \nu, \sigma_{i}\right)
$$

Then, if $\log \Lambda_{n}^{F} \geq \log A^{F}$, dismiss the conjunction; if $\log \Lambda_{n}^{F} \leq \log B^{F}$, consider performing a CAM; otherwise, if $\log B^{F}<\log \Lambda_{n}^{F}<\log A^{F}$, attempt to obtain another observation. If it is not possible to obtain another observation, then it is presumably preferable to perform the CAM, with the understanding that the false alarm probability will be increased. 

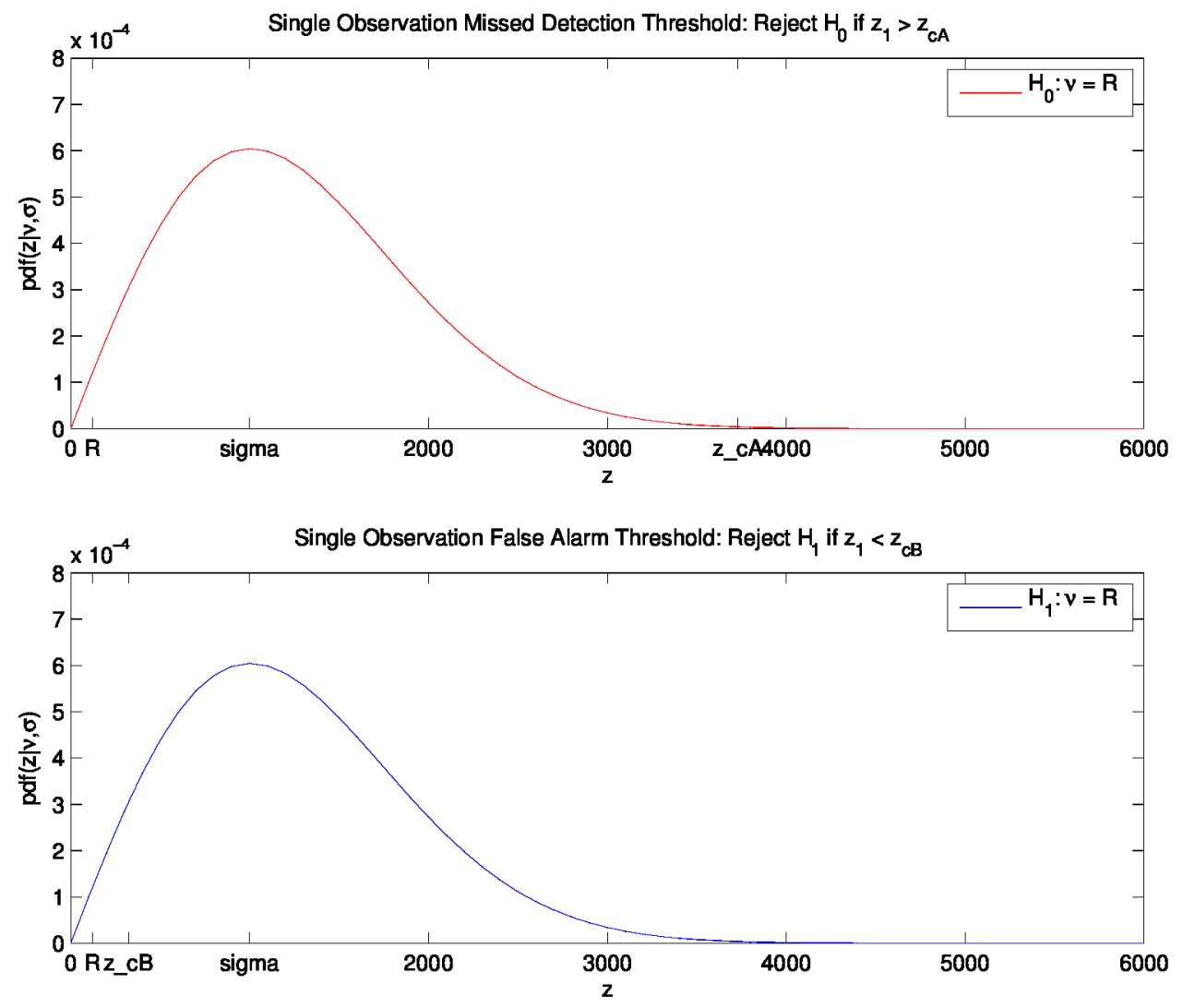

Figure 5. Critical measurement thresholds for frequentist Wald test. 


\section{Bayesian Approach}

Suppose there exists a prior density for the re-scaled true miss distance, $f(\nu)$. This may be the case, for example, if the conjunction occurs between two formation flying spacecraft whose trajectories have been designed to approach to within a specified distance, $\nu_{*}$, which has some standard deviation $\sigma_{*}$, reflecting the precision to which the formation can maneuver to achieve the design goal. Then, Bayes' Rule rule says that the probability density for a single measurement being $z_{1}$ conditioned on the distance of closest approach being less than or equal to $R$ is

$$
f\left(z_{1} \mid \nu \leq R\right)=\frac{f\left(z=z_{1}, \nu \leq R\right)}{\operatorname{Pr}(\nu \leq R)}=\frac{\int_{0}^{R} \operatorname{ric}\left(z_{1} \mid \nu, \sigma_{1}\right) f(\nu) \mathrm{d} \nu}{\int_{0}^{R} f(\nu) \mathrm{d} \nu}
$$

The probability density conditioned on the distance of closest approach being greater than $R$ is similarly found to be

$$
f\left(z_{1} \mid \nu>R\right)=\frac{f\left(z=z_{1}, \nu>R\right)}{\operatorname{Pr}(\nu>R))}=\frac{\int_{R}^{\infty} \operatorname{ric}\left(z_{1} \mid \nu, \sigma_{1}\right) f(\nu) \mathrm{d} \nu}{\int_{R}^{\infty} f(\nu) \mathrm{d} \nu}
$$

The integrals in Eqs. 25 and 26 must be evaluated numerically, and the infinite upper limits can be problematical. One way to make the integrals tractable is to assume that the prior density is also Rician,

$$
f(\nu)=\operatorname{ric}\left(\nu \mid \nu_{*}, \sigma_{*}\right)
$$

Figure 6 illustrates a particular example, and should be compared with Figure 4.

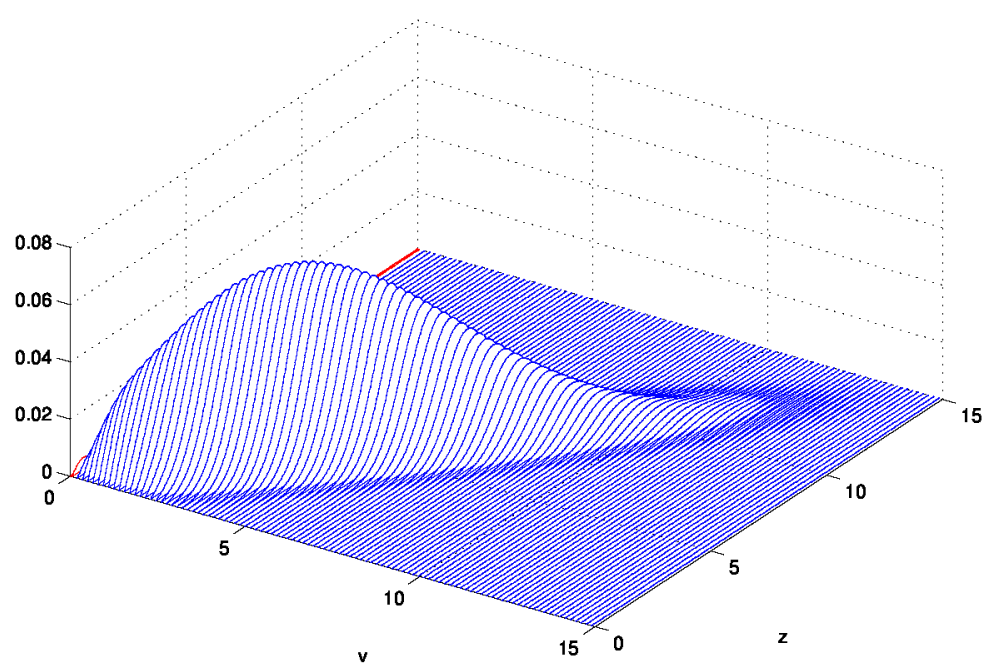

Figure 6 Convolution of the families of densities shown in Figure 4 with a prior Rician density. Here, the prior re-scaled miss distance is $\nu_{*}=3000$, and the corresponding $\sigma_{*}=3000$, and the plot has been scaled by 1:1000 to clarify the labels.

With this assumption, the integral in the denominator of Eq. 26 can be found using Marcum's $Q_{1}$ function. ${ }^{7}$ The numerator can be expressed as the integral over all $\nu$ minus the integral from 0 to 
$R$, and Eq. 6.633.4 in Gradshteyn and Ryzhik ${ }^{8}$ provides an analytic solution to the infinite integral. With the substitution [9, Eq. 9.6.3] $J_{0}(i z)=I_{0}(z)$, it gives

$$
\int_{0}^{\infty} x \exp \left(-\alpha x^{2}\right) I_{0}(\beta x) I_{0}(\gamma x) \mathrm{d} x=\frac{1}{2 \alpha} \exp \left(\frac{\beta^{2}+\gamma^{2}}{4 \alpha}\right) I_{0}\left(\frac{\beta \gamma}{2 \alpha}\right)
$$

Straightforward algebra then leads to the intuitively pleasing result that the convolution of two Rician densities is a Rician density with variance equal to the sum of the variances of the component densities, and in particular

$$
\int_{0}^{\infty} \operatorname{ric}\left(z_{1} \mid \nu, \sigma_{1}\right) \operatorname{ric}\left(\nu \mid \nu_{*}, \sigma_{*}\right) \mathrm{d} \nu=\operatorname{ric}\left(z_{1} \mid \nu_{*}, \sqrt{\sigma_{*}^{2}+\sigma_{1}^{2}}\right)
$$

The ratio of the probabilities of Eqs. 26 and 25 then gives the likelihood ratio for a single measurement as

$$
\Lambda_{1}^{B}=\frac{f\left(z_{1} \mid \nu>R\right)}{f\left(z_{1} \mid \nu \leq R\right)}=\left[\frac{\operatorname{ric}\left(z_{1} \mid \nu_{*}, \sqrt{\sigma_{*}^{2}+\sigma_{1}^{2}}\right)}{\int_{0}^{R} \operatorname{ric}\left(z_{1} \mid \nu, \sigma_{1}\right) \operatorname{ric}\left(\nu \mid \nu_{*}, \sigma_{*}\right) \mathrm{d} \nu}-1\right] \frac{\int_{0}^{R} \operatorname{ric}\left(\nu \mid \nu_{*}, \sigma_{*}\right) \mathrm{d} \nu}{\int_{0}^{\infty} \operatorname{ric}\left(\nu \mid \nu_{*}, \sigma_{*}\right) \mathrm{d} \nu}
$$

Assuming the measurements are independent gives the likelihood ratio for the set of $n$ measurements as

$$
\Lambda_{n}^{B}=\frac{f\left(\mathcal{Z}_{n} \mid \nu>R\right)}{f\left(Z_{n} \mid \nu \leq R\right)}=\left[\frac{\int_{0}^{R} \operatorname{ric}\left(\nu \mid \nu_{*}, \sigma_{*}\right) \mathrm{d} \nu}{\int_{0}^{\infty} \operatorname{ric}\left(\nu \mid \nu_{*}, \sigma_{*}\right) \mathrm{d} \nu}\right]^{n} \prod_{i=1}^{n}\left[\frac{\operatorname{ric}\left(z_{i} \mid \nu_{*}, \sqrt{\sigma_{*}^{2}+\sigma_{i}^{2}}\right)}{\int_{0}^{R} \operatorname{ric}\left(z_{i} \mid \nu, \sigma_{i}\right) \operatorname{ric}\left(\nu \mid \nu_{*}, \sigma_{*}\right) \mathrm{d} \nu}-1\right]
$$

Finding the decision ratios $A^{B}$ and $B^{B}$ in the Bayesian approach parallels the frequentist approach in basing these values on the results of a single measurement. We choose $z_{A}$ to give the desired probability of a missed detection,

$$
\begin{aligned}
P_{m d}=\operatorname{Pr}\left(z>z_{A} \mid \nu \leq R\right) & =\frac{\int_{z_{A}}^{\infty} \int_{0}^{R} \operatorname{ric}\left(z \mid \nu, \sigma_{1}\right) \operatorname{ric}\left(\nu \mid \nu_{*}, \sigma_{*}\right) \mathrm{d} \nu \mathrm{d} z}{\int_{0}^{R} \operatorname{ric}\left(\nu \mid \nu_{*}, \sigma_{*}\right) \mathrm{d} \nu} \\
& =1-\frac{\int_{0}^{z_{A}} \int_{0}^{R} \operatorname{ric}\left(z \mid \nu, \sigma_{1}\right) \operatorname{ric}\left(\nu \mid \nu_{*}, \sigma_{*}\right) \mathrm{d} \nu \mathrm{d} z}{\int_{0}^{R} \operatorname{ric}\left(\nu \mid \nu_{*}, \sigma_{*}\right) \mathrm{d} \nu}
\end{aligned}
$$

and choose $z_{B}$ to give the desired probability of false alarm,

$$
\begin{aligned}
P_{f a}=\operatorname{Pr}\left(z<z_{B} \mid \nu>R\right) & =\frac{\int_{0}^{z_{B}} \int_{R}^{\infty} \operatorname{ric}\left(z \mid \nu, \sigma_{1}\right) \operatorname{ric}\left(\nu \mid \nu_{*}, \sigma_{*}\right) \mathrm{d} \nu \mathrm{d} z}{\int_{R}^{\infty} \operatorname{ric}\left(\nu \mid \nu_{*}, \sigma_{*}\right) \mathrm{d} \nu} \\
& =\frac{\int_{0}^{z_{B}} \operatorname{ric}\left(z \mid \nu_{*}, \sqrt{\sigma_{*}^{2}+\sigma_{1}^{2}}\right) \mathrm{d} z-\int_{0}^{z_{B}} \int_{0}^{R} \operatorname{ric}\left(z \mid \nu, \sigma_{1}\right) \operatorname{ric}\left(\nu \mid \nu_{*}, \sigma_{*}\right) \mathrm{d} \nu \mathrm{d} z}{\int_{R}^{\infty} \operatorname{ric}\left(\nu \mid \nu_{*}, \sigma_{*}\right) \mathrm{d} \nu} .
\end{aligned}
$$

Then, we substitute $z_{A}$ and $z_{B}$ into Eq. 30 to find $A^{B}$ and $B^{B}$ respectively. 


\section{EXAMPLE PROBLEM}

\section{Example Problem Description}

To test our approach, we developed a static example problem based on the MMS close approach described previously. For this example, the re-scaled hard-body radius, $R$, is 37 units, corresponding to $120 \mathrm{~m}$ for the particular close encounter geometry shown in Figure 1. The re-scaled uncertainty, $\sigma_{1}$, is 377 units, which corresponds to the minor axis of the unscaled 377 by 3882 meter conjunction plane error ellipse shown in Figure 1. We assume that a small number of additional observations of the miss distance would be available subsequent to the prediction in Figure 1. These observations could be more accurate, if they were based on additional tracking from assets other than those used for the initial observation; in any case they would be more recent as well, hence reducing error due to propagation. To approximate this decrease in uncertainty, we have assumed $\sigma_{i}=\sigma_{1} / i$ for this example. To determine the decision tresholds $A$ and $B$, we used desired missed detection and false alarm probabilities of $1 / 1000$ and $1 / 20$, respectively.

To test the frequentist approach, we generated 3000 samples of the true re-scaled miss distance from a uniform distribution spanning $\left[0,2 \sqrt{3} \sigma_{1}\right]$. For each case, we generated a sequence of Rician samples, $z_{i}$, using the true $\nu$ for that case. For the prescribed missed detection and false alarm probabilities, the resulting thresholds were $\log _{10}\left(A^{F}\right)=2.04$ and $\log _{10}\left(B^{F}\right)=-.002$.

To test the Bayesisan approach, we generated 3000 samples of the true re-scaled miss distance from a Rician distribution assuming $\nu_{*}=3000$ and $\sigma_{*}=3000$. The former is motivated by the unscaled design limit for close approaches for MMS of $6 \mathrm{~km}$. As for the frequentist example, for each case, we generated a sequence of Rician samples, $z_{i}$, using the true $\nu$ for that case. For the prescribed missed detection and false alarm probabilities, the resulting thresholds were $A^{B}=$ $42.8=10^{1.63}$ and $B^{B}=0.136=10^{-.866}$.

\section{Example Problem Results and Discussion}

We repeated the 3000-case trials several times to verify repeatable results. We determined from these trials that the frequentist approach reliably produces a false alarm probability of $15 \pm 1 \%$, and we never observed a missed detection. The average number of measurements required to resolve a decision, inclusive of the first observation, was two to three. In cases where the true $\nu$ was very close to $R$, the frequentist approach often required many more observations; as many as 50 were observed. In all cases, the false alarms occurred when $\nu \approx R$.

Because the frequentist approach does not assume a prior density for $\nu$, when $\nu \approx R$, its maximum likelihood estimation produces estimates of $\nu$ under both hypotheses that are close to $\nu=R$. Thus, it appears to be difficult for the frequentist approach to distinguish between the hypotheses. We believe this is the reason why the test fails to come closer to the desired false alarm specification of $1 / 20$. Note that the limits shown by Eq. 14 apply to the actual false alarm and missed detection probabilities for the test, not the desired probabilities used to derive $A$ and $B$, and hence they are trivially satisfied by the results of our trials.

For the Bayesian approach, we also observed zero missed detections in 3000 trials, and we observed a false alarm rate of $2.8 \%$. The average number of measurements required to resolve a decision was just over one, and the maximum in 3000 trials was four.

Because the Bayesian approach has access to prior information, it appears that it is better able to distinguish between the two hypotheses. However, since the samples of the true $\nu$ were drawn 
from a Rician centered at 3000 units, we expect that many fewer observations near $R$ occurred. Therefore, we re-ran the Bayesian case with 50,000 trials. Once again, we observed a false alarm rate of $2.8 \%$, and a missed detection rate of 0 .

\section{SUMMARY}

In this paper, we stated the case that the CAM decision problem might beneficially be viewed as a problem of decisions. We then applied a well-known decision procedure, Wald's SPRT, to the CAM decision problem. When prior densities are available, we described a Bayesian approach to resolving the CAM decision problem into two simple hypotheses that applicable to the SPRT. For other cases, we described a frequentist approach that does not require prior information. We found from our examples, that, due to the nature of its reduction to Rician densities, the CAM decision problem is a challenging application of Wald's theory with the frequentist approach, but that the Bayesian method works quite well. We believe our results show that our approaches for applying the SPRT to the CAM decision problem have the potential to help decision makers better understand the trades they face when confronted by a looming CAM decision.

In future work, we hope to further validate and refine our method using additional MMS close approach simulations, including if possible CAM's to avoid other resident space objects. We also plan to examine the sensitivity of each of our approaches to different prior densities, to the assumption of independence of the measurements, and to relaxation of the some of the assumptions inherent in the reduction of the full CAM decision problem to the evaluation of Rician densities.

\section{REFERENCES}

[1] A. Wald, Sequential Analysis. Dover Publications, 2004.

[2] J. L. Foster, Jr. and H. S. Estes, "A Parametric Analysis of Orbital Debris Collision Probability and Maneuver Rate for Space Vehicles," Tech. Rep. JSC-25898, NASA Johnson Space Center, Houston, TX, 1992.

[3] M. R. Akella and K. T. Alfriend, "Probability of Collision Between Space Objects," Journal of Guidance, Control and Dynamics, Vol. 23, September-October 2000, pp. 769-772.

[4] K. Chan, "Improved Analytical Expressions for Computing Spacecraft Collision Probabilities," Space Flight Mechanics 2003, Univelt, 2003.

[5] A. Papoulis, Probability, Random Variables, and Stochastic Processes, ch. 7, pp. 194-196. McGraw-Hill, 2nd ed., 1965.

[6] M. H. DeGroot, Probability and Statistics. Addison-Wesley, 1975.

[7] J. I. Marcum, “Table of $Q$ Functions," US Air Force RAND Research Memorandum M-339, Rand Corporation, Santa Monica, CA, Jan. 11950.

[8] I. S. Gradshteyn and I. M. Ryzhik, Table of Integrals, Series, and Products. New York: Academic Press, 1965.

[9] M. Abramowitz and I. A. Stegun, Handbook of Mathematical Functions. New York: Dover, 1965. 